\title{
ENAMEL SURFACE ROUGHNESS ASSESSMENT AFTER DEBONDING, EMPLOYING THREE DIFFERENT REMOVAL METHODS.
}

1. BDS, Fellowship Training in Orthodontics

Associate Orthodontist Department of Orthodontists Ihsan Mumtaz Hospital, Lahore.

2. BDS, M Orth RCS ED (UK) Consultant Orthodontist Department of Orthodontists, Ihsan Mumtaz Hospital, Lahore.

3. BDS, M.Phil (Physiology) Assistant Professor Central Park Medical College, Lahore.

4. BDS, M Orth RCS ED (UK) Assistant Professor

Frontier Medical \& Dental College, Abbottabad.

5. BDS, M.Phil.

Assistant Professor \& HOD Department of Oral Biology Avicenna Medical \& Dental College, Lahore.

6. BDS, Fellowship Training in Orthodontics Senior Demonstrator Department of Orthodontists Shareef Medical \& Dental College, Lahore.

Correspondence Address:

Dr. Uzma ljaz

71-E, Maulana Shaukat Ali Road, Johar Town, Lahore.

17-b, St- 6, Shah Jamal, Lahore. wasp8@hotmail.com

Article received on:

20/06/2019

Accepted for publication: 20/08/2019

Received after proof reading: $28 / 08 / 2019$

\begin{abstract}
Uzma ljaz', Kashif Haroon², Saad Haroon ${ }^{3}$, Taimoor Khan', Raheela Yasmin ${ }^{5}$, Rashid Mehmood
\end{abstract}
ABSTRACT: The search for an efficient and safe resin removal method after debonding has resulted in the introduction of a wide array of instruments and techniques. Previously, safety of rotary instruments was limited to inspecting the surface under a scanning electron microscope that lacks a quantitative scale. In this study, comparative assessment of the enamel roughness was done quantitavely using surface profilometer. Objectives: To evaluate quantitatively the enamel surface roughness following debonding using three different resin removal methods (composite removing pliers, ultrasonic scaler and low speed Tungsten Carbide bur). Study Design: Prospective study. Setting: Orthodontic clinic of Ihsan Mumtaz Hospital Lahore and PCSIR (Lahore). Period: 6 months from June 2018 to December 2018. Material and Methods: Ninety, healthy extracted maxillary premolars were taken and subjected to profilometric analysis to register four roughness parameters. Brackets were bonded and all specimens were immersed in distilled water for one week. After debonding, teeth were randomly divided into three groups and subjected to different resin removal methods. A second roughness recording was taken and compared with roughness at baseline interval. Enamel surface roughness with three resin removal methods were also compared with one other. Data Analysis: SPSS Version 20.0 was used. Paired $t$ test was applied within three groups separately to establish the comparison between the enamel surface roughnesses at baseline. One way ANOVA was used to establish the comparison of increase in enamel surface roughness among three study groups compared using different resin removal methods (slow speed tungsten carbide bur, ultrasonic scaler and composite removing pliers). Results: Slow speed tungsten carbide bur created the least increase in enamel surface roughness while ultra-sonic scaler had the most elevated values. Conclusion: Enamel surface roughness following debonding can be minimized with the use of tungsten carbide bur for resin removal in a slow speed hand piece.

Key words: Debonding, Enamel Surface Roughness, Resin Removal.

Article Citation: ljaz U, Haroon K, Haroon S, Khan T, Yasmin R, Mehmood R. Enamel surface roughness assessment after debonding, employing three different removal methods. Professional Med J 2019; 26(9):1511-1517.

DOI: $10.29309 / \mathrm{TPMJ} / 2019.26 .09 .3841$

\section{INTRODUCTION}

At the consummation of orthodontic treatment, one of the clinician's main goal is to bring the enamel surface close to its initial state as possible. While putting on brace, taking them off and during adhesive removal, minimal enamel damage should be the goal. ${ }^{1}$ Enamel is cleaned by rubber cups or brushes by some doctors before etching, etchant also demineralizes the enamel and when brackets are pulled off of the tooth surface at debonding, especially ceramic brackets, there's a certain risk of enamel fracture; these all procedures are linked to enamel damage. $^{2}$
The outer most layer of enamel contains high Fluoride and mineral content that leads to its hardness. Damaging this layer, makes enamel prone and less resistant to dissolution by acid by-products of plaque and more chances of decalcification. These enamel surface changes may occur during pre-etching cleaning, during phosphoric acid etching and mainly during debonding and surface clean up especially with rotary instruments. ${ }^{3,4}$

Researchers have been looking for competent and proficient methods for removing adhesive 
resin after debonding. This has led to prolusion of different sorts and types of instruments and methods for resin removal. The protracted list of procedures and instruments are available now. Including manual methods with the use of debonding pliers or scalers, different shapes of Tungsten Carbide burs installed in high or low speed hand pieces and polishing pastes containing Zirconia or slurry pumice. ${ }^{3-6}$ Recent ways involve laser methods like that of Carbon dioxide and $\mathrm{Nd}$ : YAG lasers which looking expensive but promising for adhesive removal over the enamel and not damaging it much. ${ }^{7}$ It was concluded that oral hygiene procedures have a mild to insignificant effect during active orthodontic treatment. ${ }^{8}$

There are plethoras oftechniques at one's disposal to remove resin from the enamel s surface after the brackets have been pulled off. But to appraise the enamel surface changes after these different debonding methods have not progressed at the same speed and stride. To check the efficacy and safeness of rotary instruments over enamel, SEM (scanning electron microscope) has been most effective to declare the conformation and topography of enamel surface..$^{9-11}$ That is why; standard microscopes are not very effective in analyzing the enamel surface topography for enamel surface amendments. Therefore in this study, comparative assessment of the enamel roughness on the quantitative scale using surface profilometer has been done.

\section{METHODOLOGY}

The prospective study was conducted at Orthodontic clinic of Ihsan Mumtaz Hospital Lahore and PCSIR (Lahore). Duration of the study was 6 months from June 2018 to December 2018.

Ninety (90) human maxillary premolars extracted for orthodontic purpose were used for this study. A verbal consent was taken from the patients about usage of the teeth in the study. Teeth were indexed and then randomized to three groups of 30 each. Sampling Technique was Nonprobability purposive sampling

\section{Inclusion Criteria}

Was extracted healthy human premolar teeth without surface enamel defects, devoid of carious lesion, no evidence of enamel cracking and restorations.

\section{Exclusion Criteria}

Was fractured teeth, hypoplastic teeth, teeth previously bonded with brackets, teeth pretreated with chemicals such as Hydrogen per oxide.

\section{Data Collection Procedure}

Ninety premolars were used in the study, which were extracted for orthodontic reasons. Their extraction was not related to this investigation, so there were no ethical issues.

The teeth were cleaned and with the help of saw, roots were removed. Teeth were placed in plaster cylinders with labial/buccal surfaces exposing for the procedure to be worked upon. Rectangular pieces of black adhesive tape were placed over tooth's middle third part of the buccal surface. For standardization, opening of $3 \mathrm{~mm}$ (round in shape) was left on the tape, so teeth can be analyzed for bonding, profilometric checkup and analysis. Teeth were coded for identification reasons, and exposed enamel parts were analyzed by the profilometer. Four roughness parameters were noted and registered before any procedure (baseline interval). The average roughness (Ra), which described the overall surface roughness, and can be defined as the arithmetic mean of all absolute distances of the roughness profile from the centre line within the measuring length. The root mean square roughness $(\mathrm{Rq})$, representing the height distribution relative to the mean line. The maximum roughness depth (Rt), which registered isolated profile features on the surface. $\mathrm{Rz}$, which described the average maximum peakto-valley height of five consecutive sampling depths.

All the teeth used in the study were etched with Phosphoric acid (37\%) and were washed and dried. Metal premolar brackets (3M/Unitek, Monrovia, California, USA) were adhered with composite adhesive. After complete sample preparation, teeth were kept in distilled water for 1 week and then the brackets were pulled off 
with the help of a debonding plier. Three groups were made by randomly dividing the specimen. In Group 1, composite removing pliers were used for removing remnants of adhesive resin. In Group 2; the adhesive remnants were removed with an ultrasonic scaler and in Group 3, Tungsten Carbide bur was used in a slow speed hand piece and the bur changed on each specimen. (Resin removal interval) a second recording was noted after removal of the resin. The depth of resin removal was seen satisfactorily by visual inspection under dental unit's light. Enamel surface roughness in microns, at baseline interval and post resin removal interval was compared and also the enamel surface roughness at post resin removal interval, using three different methods for resin removal was compared with one another.

\section{DATA ANALYSIS}

SPSS Version 20.0 was used to analyze the data. Paired t test was applied within three groups separately to establish the comparison between the enamel surface roughnesses at baseline. One way ANOVA was used to establish the comparison of increase in enamel surface roughness among three study groups compared using different resin removal methods (composite removing pliers, ultra sonic scaler and Tungsten Carbide bur in a slow speed hand piece).

\section{RESULTS}

In Figure-1 (Table-I), with composite removing pliers as resin removal method, all the roughness variables showed elevated values after resin removal. Baseline value of mean average roughness ( $\mathrm{Ra})$ was $3.4 \mu \mathrm{m}$ with a standard deviation of $0.62 \mu \mathrm{m}$. After resin removal, mean average roughness increased to $4.64 \mu \mathrm{m}$ with a standard deviation of $0.88 \mu \mathrm{m}$. Which is clearly a significant difference $(p<0.001)$. At baseline interval, average maximum height of the profile (Rz) was $15.10 \mu \mathrm{m}$ with a standard deviation of $1.11 \mu \mathrm{m}$ and it increased to $16.53 \mu \mathrm{m}$ with a standard deviation of $1.37 \mu \mathrm{m}$ after resin removal which showed a significant difference $(p<0.001)$. Mean root square roughness $(\mathrm{Rq})$ at baseline interval also differed having mean of $4.2 \mu \mathrm{m}$ with a standard deviation of $0.58 \mu \mathrm{m}$ from the after resin removal values having mean of $5.46 \mu \mathrm{m}$ with a standard deviation of $0.97 \mu \mathrm{m}(\mathrm{p}<0.001)$. The mean maximum roughness depth significantly increased from $14.35 \mu \mathrm{m}$ to $15.59 \mu \mathrm{m}$ after resin removal $(p<0.0001)$.

In Figure-2 (Table-II), when resin was removed with ultrasonic scaler, the mean average roughness increased from $3.74 \mu \mathrm{m}$ to $6.32 \mu \mathrm{m}$ $(p<0.001)$. The mean average maximum height of the profile increased from $15.27 \mu \mathrm{m}$ to $17.78 \mu \mathrm{m}$ after resin removal $(p<0.001)$. The root mean square roughness increased from $4.39 \mu \mathrm{m}$ to $6.84 \mu \mathrm{m}(p<0.001)$, while the mean maximum roughness depth increased from $14.31 \mu \mathrm{m}$ to $17.05 \mu \mathrm{m}(p<0.001)$ after resin removal.

In Figure-3 (Table-III), mean and standard deviation of roughness variables before and after resin removal when slow speed tungsten carbide bur was used for resin removal. The average roughness increased from $3.28 \mu \mathrm{m}$ to $3.85 \mu \mathrm{m}(p<0.001)$. The average maximum height of profile increased from $14.81 \mu \mathrm{m}$ to $15.73 \mu \mathrm{m}$ $(p<0.001)$. The root mean square roughness increased from $4.47 \mu \mathrm{m}$ to $5.38 \mu \mathrm{m}(p<0.001)$. The mean maximum roughness depth increased from $13.10 \mu \mathrm{m}$ to $14.48 \mu \mathrm{m}(\mathrm{p}<0.001)$.

As can be seen from Tables-I,II and III, there is a significant difference between roughness at baseline interval and at post resin removal interval within three groups with $\mathrm{p}$-value $<0.001$.

One way ANOVA was used to establish the comparison of increase in enamel surface roughness among three study groups compared using different resin removal methods (slow speed tungsten carbide bur, ultrasonic scaler and composite removing pliers). As can be seen from Table-IV and Figure-4, there is a significant difference among the three groups compared with $\mathrm{p}$-value $<0.001$.

\section{DISCUSSION}

The results of this investigation showed that all Roughness variables presented higher values at the adhesive removal interval, with the use of three resin removal methods. The ultrasonic scaler created the highest values. ${ }^{12}$ 


\begin{tabular}{|l|c|c|c|c|}
\hline $\begin{array}{c}\text { Roughness } \\
\text { Variables }(\boldsymbol{\mu m})\end{array}$ & $\begin{array}{c}\text { At base line interval } \\
\mathbf{n}=\mathbf{3 0}\end{array}$ & $\begin{array}{c}\text { After resin removal } \\
\mathbf{n = 3 0}\end{array}$ & $\mathbf{t}$ & P-Value \\
\hline & Mean \pm SD & Mean \pm SD & & \\
\hline $\mathrm{Ra}$ & $3.40 \pm 0.62$ & $4.64 \pm 0.88$ & 16.32 & $<0.001$ \\
\hline $\mathrm{Rz}$ & $15.10 \pm 1.11$ & $16.53 \pm 1.37$ & 13.60 & $<0.001$ \\
\hline $\mathrm{Rq}$ & $4.20 \pm 0.58$ & $5.46 \pm 0.97$ & 12.69 & $<0.001$ \\
\hline $\mathrm{Rt}$ & $14.35 \pm 0.95$ & $15.59 \pm 1.01$ & 12.98 & $<0.001$ \\
\hline
\end{tabular}

Table-I. Roughness of enamel surface at base line interval and after resin removal using composite removing plier as resin removal method

\begin{tabular}{|l|c|c|c|c|}
\hline $\begin{array}{c}\text { Roughness } \\
\text { Variables }(\boldsymbol{\mu m})\end{array}$ & $\begin{array}{c}\text { At base line interval } \\
\mathbf{n = 3 0}\end{array}$ & $\begin{array}{c}\text { After resin removal } \\
\mathbf{n = 3 0}\end{array}$ & $\mathbf{t}$ & P-Value \\
\hline & Mean \pm SD & Mean \pm SD & & \\
\hline $\mathrm{Ra}$ & $3.74 \pm 0.48$ & $6.32 \pm 0.92$ & 21.28 & $<0.001$ \\
\hline $\mathrm{Rz}$ & $15.27 \pm 1.38$ & $17.78 \pm 1.47$ & 29.17 & $<0.001$ \\
\hline $\mathrm{Rq}$ & $4.39 \pm 0.53$ & $6.84 \pm 1.02$ & 18.04 & $<0.001$ \\
\hline $\mathrm{Rt}$ & $14.31 \pm 1.35$ & $17.05 \pm 1.48$ & 29.12 & $<0.001$ \\
\hline
\end{tabular}

Table-II. Roughness of enamel surface at base line interval and after resin removal using ultra-sonic scaler as resin removal method:

\begin{tabular}{|l|c|c|c|c|}
\hline $\begin{array}{c}\text { Roughness } \\
\text { Variables }(\boldsymbol{\mu m})\end{array}$ & $\begin{array}{c}\text { At base line interval } \\
\mathbf{n}=\mathbf{3 0}\end{array}$ & $\begin{array}{c}\text { After resin removal } \\
\mathbf{n = 3 0}\end{array}$ & $\mathbf{t}$ & P-Value \\
\hline & Mean \pm SD & Mean \pm SD & & \\
\hline $\mathrm{Ra}$ & $3.28 \pm 0.45$ & $3.85 \pm 0.57$ & 9.44 & $<0.001$ \\
\hline $\mathrm{Rz}$ & $14.81 \pm 1.38$ & $15.73 \pm 1.47$ & 8.66 & $<0.001$ \\
\hline $\mathrm{Rq}$ & $4.47 \pm 0.49$ & $5.38 \pm 0.66$ & 10.68 & $<0.001$ \\
\hline $\mathrm{Rt}$ & $13.10 \pm 1.25$ & $14.48 \pm 1.33$ & 10.40 & $<0.001$ \\
\hline
\end{tabular}

Table-III. Roughness of enamel surface at base line interval and after resin removal using tungsten carbide bur as resin removal method

\begin{tabular}{|c|c|c|c|c|}
\hline & \multicolumn{3}{|c|}{$\begin{array}{c}\text { Increase in Roughness variables } \\
\text { Mean } \pm \text { Std. dev. }\end{array}$} & \multirow[t]{2}{*}{ P-Value } \\
\hline & $\underset{n=30}{\text { Group I }}$ & $\begin{array}{c}\text { Group II } \\
n=30\end{array}$ & $\begin{array}{c}\text { Group III } \\
n=30\end{array}$ & \\
\hline $\mathrm{nRa}$ & $1.24 \pm 0.41$ & $2.58 \pm 0.66$ & $0.57 \pm 0.33$ & $<0.001$ \\
\hline $\mathrm{nRz}$ & $1.42 \pm 0.57$ & $2.50 \pm 0.47$ & $0.92 \pm 0.58$ & $<0.001$ \\
\hline $\mathrm{nRq}$ & $1.26 \pm 0.54$ & $2.45 \pm 0.74$ & $0.91 \pm 0.47$ & $<0.001$ \\
\hline nRt & $1.24 \pm 0.52$ & $2.74 \pm 0.51$ & $1.38 \pm 0.73$ & $<0.001$ \\
\hline
\end{tabular}

Table-IV. One way analysis of variance by groups

Manual removal with composite removing plier create less enamel roughness ${ }^{13}$ while least values of roughness variables with slow speed tungsten carbide bur at post resin removal support its use as preferable method. ${ }^{14}$

The result was in accordance with the previous study conducted by Theodore Eliades et al in $2004 .{ }^{15}$ They used 30 human premolar teeth and the best method of adhesive removal was
Tungsten Carbide bur as it produced less enamel roughness than diamond bur.

Ingrid Hosein et $\mathrm{al}^{16}$ in their study of enamel loss during bonding, debonding and clean up with use of a self etching primer also concluded that least enamel loss occurred with the use of slowspeed tungsten carbide bur. 
Roughness of enamel surface at base line interval and after resin removal using composite removing plier as resin removal method

20

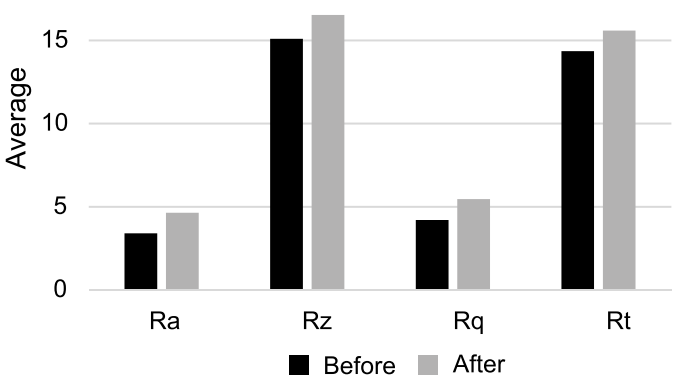

Roughness Variables $(\mu \mathrm{m})$

Figure-1

Roughness of Enamel surface at base line interval and after resin removal using tungsten carbide bur as resin removal method

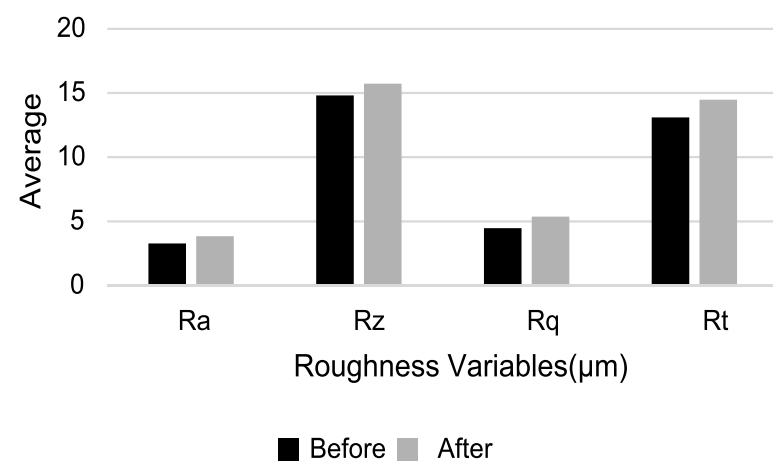

Figure-3

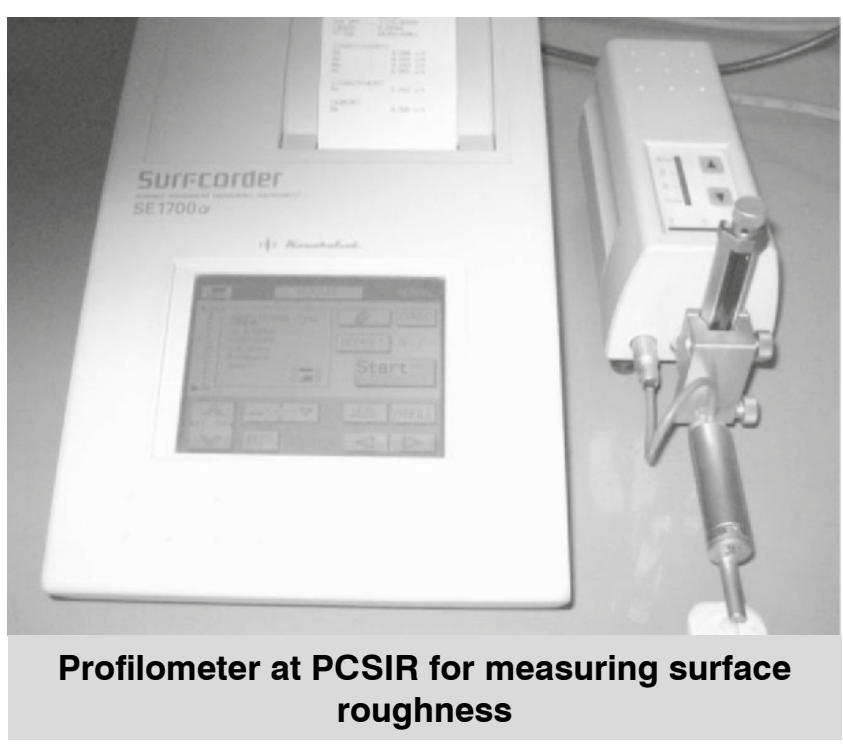

Roughness of enamel surface at base line interval and after resin removal using ultrasonic scaler as resin removal method

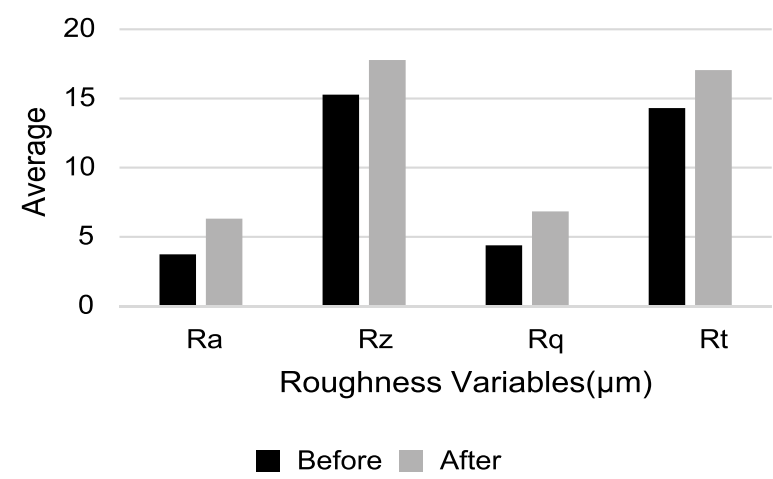

Figure-2

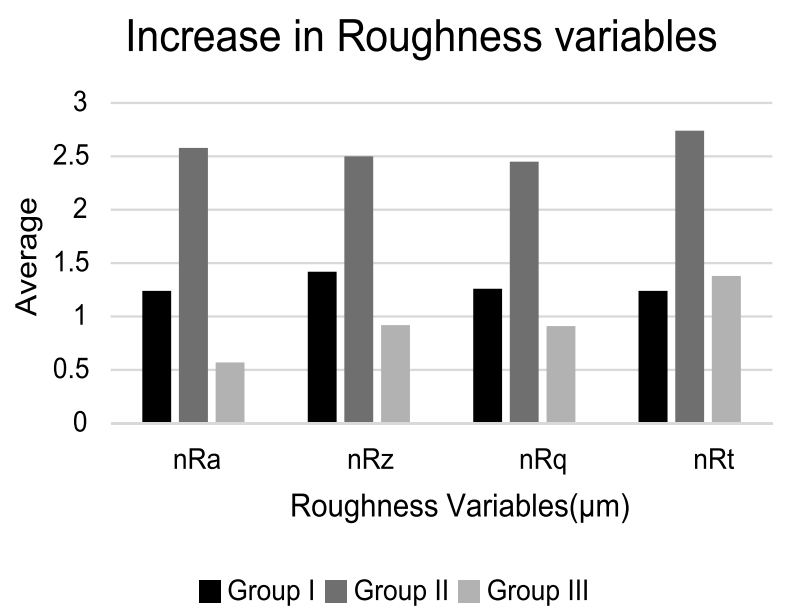

Figure-4

In 2003, Martina Miksic et $\mathrm{al}^{17}$ in their study did the quantitative analysis of Enamel surface after resin removal. The study was carried out on 30 human premolars. The best enamel surface appearance was determined after using the tungsten carbide bar, which is considered the method which causes the least damage to the enamel surface.

The amount of enamel removed during bonding and debonding is related to several factors, including the instruments used for prophylaxis and debonding and the type of adhesive resin used. ${ }^{18,3-5}$

During prophylaxis initially, more enamel gets abraded away with a bristle brush as compared 
to a rubber cup. ${ }^{19}$ Browny and Way ${ }^{20}$ discovered that highly filled resins caused more enamel loss than unfilled resins when the resin is removed as their adequate removal generally requires rotary instrumentation.

\section{CONCLUSION}

The results of our in-vitro investigation show that residual composite on the tooth surface can be removed with lesser enamel alteration with meticulous use of a tungsten-carbide bur in slow speed hand piece.

\section{Limitations of the Study}

It should be kept in mind that profilometry does not tell us about the composition of the material being scrutinized. Thus this subject should be further investigated.

\section{Acknowledgements}

The authors acknowledge the efforts of $\mathrm{Mr}$ Hammad (Engineer) at PCSIR in helping us do the tests with the profilometer. We would like to thank Mr Mujahid (accounting and management department personnel at Ihsan Mumtaz Hospital) in printing and logistics of writing of this manuscript.

No financial support was claimed.

\section{Conflict of Interest}

The authors declare no conflict of interest for this work.

Copyright@ 20 Aug, 2019.

\section{REFERENCES}

1. Hosein I, Scherriff M, Ireland AJ. Enamel loss during bonding, debonding and clean-up with use of a self -etching primer. Am J Orthod Dentofacial Orthop 2004; 126:717-24.

2. Eminkahyagil N, Arman A, Cetinsahin A, Karabulut E. Effect of Resin-removal methods on enamel and shear bond strength of rebonded brackets. Angle Orthod. 2006; 76: 314-21.

3. Efficacy of auxiliary devices for removal of fluorescent residue after bracket debonding. Rocha RS, Salomão FM, Machado LS, Sundfeld RH and Fagundes TC. The Angle Orthodontist 2017 87:3, 440-47.
4. Comparison of characteristics of de-bonded enamel surface after various resin removal methods using atomic force microscopy: An in vitro study: Balachandran $\mathrm{H}$, Varghese ST, Krishnaraj R, S Sreenivas, Jose LK and V R Joshy. Journal of International Oral Health 2016; 8(5):595-600.

5. Evaluation of enamel surface roughness after orthodontic bracket debonding with atomic force microscopy. Mohebia S, Shafieeb H andAmelic N. Am J Orthod Dentofac Orthop 2017 151:3, 521-27.

6. In-vivo evaluation of the surface roughness and morphology of enamel after bracket removal and polishing by different techniques. Faria-Júnior EM, Guiraldo RD, Berger SB, Correr AB et al. Am J Orthod Dentofac Orthop 2015 147:3, 324-29.

7. Eliades T, Gioka C, Eliades G and Makou M. Enamel surface roughness following using debonding two resin grinding methods. Eur J Orthod. 2004; 26: 333-8.

8. Rumi $H$. Oral hygiene evaluation in orthodontic practice. Pak Oral Dent J 2002; 22: 31-8.

9. Hammad N, Salama F. Enamel surface and resin tag morphology: SEM Study. Pak Oral Dent J 2001; 10:1724.

10. Sigilião, Freitas LC, Marquezan, Mariana, Elias and Nelson $C$ et al. Efficiency of different protocols for enamel clean-up after bracket debonding: An in vitro study. Dental Press Journal of Orthodontics 2015, 20(5), 78-85.

11. Epidemiological survey of different clinical techniques of orthodontic bracket debonding and enamel polishing. Sfondrini MF, Scribante A, Fraticelli D, Roncallo S and Gandini.JP Orthod Sci. 2015 Oct-Dec; 4(4): 123-27.

12. Arora S, Lamba AK, Faraz F, Tandon S, Ahad A. Evaluation of the effects of Er,Cr: YSGG Laser, Ultrasonic scaler and curette on root surface profile using surface analyser and scanning electron microscope: An in vitro study. J Lasers Med Sci. 2016; $7(4): 243-49$.

13. Mhatre AC, Tandur AP, Reddy SS, Karunakara BC and Baswaraj $H$. Enamel surface evaluation after removal of orthodontic composite remnants by intraoral sandblasting technique and carbide bur technique: A three-dimensional surface profilometry and scanning electron microscopic study. Journal of international oral health: 2015; 7. 34-9.

14. Tufekci E, Merrill TE, Pintado MR, Beyer JP and Brantley WA. Enamel loss associated with orthodontic adhesive removal on teeth with white spot lesions: An in vitro study. Am J Orthod Dentofac Orthop 2004; 125:733-40. 
15. Eliades T, Gioka C, Eliades G and Makou M. Enamel surface roughness following debonding using two resin grinding methods. Eur J Orthod 2004; 26:333-8.

16. Hosein I, Scherriff $M$ and Ireland AJ. Enamel loss during bonding, debonding and clean-up with use of a self -etching primer. Am J Orthod Dentofac Orthop 2004; 126:717-24.

17. Miksic M, Slaj M and Mestrovic S. Qualitative analysis of enamel surface after removal of composite. Am J Orthod Dentofac Orthop 2003: 37: 247-50.
18. Tonetto MR, Frizzera F, Porto TS et al. Methods for removal of resin remaining after debonding of orthodontic brackets: A literature review. Journal of dental research and review 2014; 1:105-7.

19. Yadav J, Raghav P, Reddy M and Ahuja NK. Evaluation of enamel loss with bonding and debonding: A study with profile projector. J Indian Orthod Soc 2017; 51:177-84.

20. Brown CR and Way DC. Enamel loss during orthodontic bonding and subsequent loss during removal of filled and unfilled adhesives. Am J Orthod 1978; 74:663-71.

\section{AUTHORSHIP AND CONTRIBUTION DECLARATION}

\begin{tabular}{|c|c|c|c|}
\hline Sr. \# & Author-s Full Name & Contribution to the paper & Author $=$ s Signature \\
\hline 1 & Uzma ljaz & $\begin{array}{l}\text { Conception and design of work, } \\
\text { data collection, critical revision and } \\
\text { final approval of the version to be } \\
\text { published. }\end{array}$ & \\
\hline 2 & Kashif Haroon & $\begin{array}{l}\text { Data collection, data interpretation } \\
\text { and drafting the artilce. }\end{array}$ & \\
\hline 3 & Saad Haroon & $\begin{array}{l}\text { Data collection, data analysis and } \\
\text { drafting the article. }\end{array}$ & \\
\hline 4 & Taimoor Khan & Data analysis and drafting the article. & \\
\hline 5 & Raheela Yasmin & Data analysis and drafting the article. & \\
\hline 6 & Rashid Mehmood & Data analysis and drafting the article. & \\
\hline
\end{tabular}

